\title{
A Fuzzy-Based Analytical Model of Dynamic Information Flows in e-SCM
}

\author{
Iraj Mahdavi \\ Mazandaran University of Science and Technology \\ E-Mail: irajarash@rediffmail.com \\ Namjae Cho \\ Hanyang University \\ E-Mail: njcho@hanyang.ac.kr \\ Shima Mohebbi \\ Mazandaran University of Science and Technology \\ E-Mail:sh_mathematical@yahoo.com
}

\begin{abstract}
Electronic marketplace (EM) has been considered as an alternative coordination mechanism to coordinate supply chain activities between suppliers and buyers. In business-to-business (B2B) electronic commerce contexts, new functional relationships have been created between suppliers and buyers in an attempt to reduce lead times and stock outs and to improve the level of customer service. In this paper we develop an ebased supply chain model and an agent for designing mass- customized online services. A new model of information flows with fuzzy logic is also suggested with a goal to achieve highly improved performance for logistical management with non-quantifiable parameters. We extend our discussion to the new role of an agent and information in supply chain activities. Information reduces the level of uncertainty which both suppliers and buyers face with. It also provides reliable and dynamic activities streamlined to supply chains of high performances. We employed heuristic approach to develop a theoretical model. Future research can analyze empirical outcomes of the application of our model.
\end{abstract}

Keywords: Information Flows, e-SCM, Fuzzy Logic, Agent 


\section{INTROUDUCTION}

Efficient and effective management of supply chain is of great importance for the success of the digital economy. A supply chain is composed of a set of entities involved in designing new products and services, procuring raw materials, transforming them into semi-finished and finished products, and delivering them to end customers. Supply chains exist both in service and manufacturing organizations. Swaminathan and Tayur (2003) describe major issues in traditional supply chain management and provide an overview of relevant analytical models to be used in the area of e-business and supply chain management. Supply chains in practice deal with multiple end-products with shared components, facilities and capacities. Traditionally, marketing, distribution, planning, manufacturing, and purchasing functions operate independently along the supply chain. These departments have their own objectives and thus are often in conflict. Over the past few decades, we have witnessed an ever- spreading globalization of the economy and thereby also of supply chains. Products are no longer produced and consumed within a single geographical area. Different parts of a product may, and often do, come from all over the world. This practice creates longer and more complex supply chains, and therefore changes requirements for the new supply chain management. Business firms are increasingly embracing integrated supply chains because they promise cost reduction, efficiency, and effective fulfillment of market demand. Rapid developments in the information and software engineering sectors have given rise to unprecedented opportunities for the integration and coordination of organizational processes and tasks by helping us overcome uncertainties in supply chain management. Electronic exchanges of information lead to the reduction of errors and to the increase of efficiency of the processes involved. When one company can access to the relevant information of other companies in the supply chain, the negative effects of uncertainty (i.e., inaccurate forecasts, higher inventory levels, etc.) can be reduced.

As global markets evolve, supply chain managers are faced with the dynamics of continuously changing markets, new global span of markets and the complexity of stressful competitive environments (Mehra and Agrawal 2003, Mehra and Inman 2004). Dealing with traditional trade offs is no longer an option. Firms begin to realize the need to optimize their supply chain strategies over a much broader base (Meredith and Roth 1998). Because supply chains extend across several functions and many organizations, 
each has its own priorities and goals (Narayanan and Raman 2004). Additional challenges are presented by the availability of electronic links with a strong potential to improve the supply chain performance (Poulymenakou and Tsironis 2003). It has become easier for organizations to observe other firms' action (Narayanan and Raman 2004) and focus on cost cutting and efficiency (Lee 2004, Liker and Choi 2004). Threats from competition and other market forces are driving changes in supply chain management (Flynn and Flynn 2005).

\section{E-SUPPLY CHAIN SYSTEM}

Both the functionality and structure of an e-supply chain system may vary from highly to loosely integrated levels. The highly integrated e-supply chain systems generally have fairly complex internal and external operations. They may have many suppliers, spread all over the world, who provide a large variety of parts and components. In order to respond to competitive challenges (e.g., maintaining customer service levels, lowering the level of inventory, etc.), an e-supply chain system should be installed to efficiently and effectively link-up complex operations. Supply Chain (SC) systems can be studied and analyzed from several viewpoints. Yet there are three major perspectives of SC systems: (a) "Material Flow", (b) "Information Flow", and (c) "Buyer-Seller Relations" (Fazel Zarandi et al. 2002). The buyer-seller relation is the major focus of interest among SC researchers and managers. Traditional approaches to buy-sell process focus on factors like the price in the buyer-seller relation. The new SC practice draws attention to quality, $\mathrm{R} \& \mathrm{D}$, cost reduction, customer satisfaction, and partnerships. In an $\mathrm{SC}$, both external and internal resources are important. The relations are not established based only on the level of price and cost.

The advent of new information systems and technologies (IS and IT) such as electronic data interchange(EDI), Internet, Intranet, and Extranet, in particular, and interorganizational communication and coordination mechanisms casts unprecedented opportunities for the integration of supply chains.

Our model of supply chain is composed of three main actors: customers or buyers who want to purchase services from suppliers; suppliers or sellers who offer the services or products; and the control/optimization service agent that facilitates the selection of business counterparts and the communication between customers and suppliers. 


\section{THE SC TRANSACTION AGENT FOR CONTROL AND OPTIMIZATION}

An agent is a technical system that supports the transactional relationships within the network of an SC. This section describes the functionality of this transaction agent (TA).TA plays the most important role in our proposed supply chain system. It mediates the interaction between customers and suppliers in an electronic marketplace. The agent is especially useful when a supply chain system has many customers and suppliers. It is helpful when the search cost is relatively high, and much of the services are delivered on the basis of trust.

\section{Agent Architecture}

The major components and functions of an agent are as follows:

a) quantitative and qualitative attributes related to customers' evaluation (see Table 1),

b) quantitative and qualitative attributes related to sellers' perception (see also Table 1),

c) preprocessing and building customer profiles,

d) preprocessing and building seller profile, and

e) preprocessing and building supplier-buyer relationship profile.

Table1 Quantitative and Qualitative Attributes Related to Buyer and Seller

\begin{tabular}{|c|c|c|}
\hline & & \\
\hline & Buyer & Seller \\
\hline Quantitative Attribute & $\begin{array}{l}\text { Price, Lead Time, } \\
\text { Transportation Cost, }\end{array}$ & $\begin{array}{c}\text { Sales Volume, Price, } \\
\text { Capacity, Product Life } \\
\text { Cycle,... }\end{array}$ \\
\hline Qualitative Attribute & $\begin{array}{c}\text { Service Level, Aesthetics, } \\
\text { Management,... }\end{array}$ & $\begin{array}{l}\text { Customer Satisfaction, } \\
\text { Technological Standard, } \\
\text { Geographical Benefit,... }\end{array}$ \\
\hline
\end{tabular}

In the next section, we will illustrate how fuzzy logic can facilitate the acquisition of rich and accurate profiles in an electronic supply chain system.

\section{Customer Profile}

The agent receives all necessary information about the quantitative and qualitative attributes related to each product from $m$ customers electronically. Then the agent assigns $n$ key attributes for each aspect of customer's perception. By obtaining all information from customers we create the vector of comprehensive key attributes as $C K=\{k 1$, $k 2, \ldots, k n\}$. The agent also designs the customer-key attributes incidence matrix as $\mathrm{CKIM}=\left[c_{\mathrm{ij}}\right]$, where $c_{\mathrm{ij}}$ represents the fuzzy value of $i^{\text {th }}$ customer on $j^{\text {th }}$ attribute for $i=$ $1,2, \ldots, m$ and $j=1,2, \ldots, n$. 
This value indicates the priority and evaluation of the customer on a special aspect of a product. On the basis of a specified threshold valuel for each key attribute, the above matrix could be converted into a binary matrix $B C K I M=\left[b c_{\mathrm{ij}}\right]$, where $b c_{\mathrm{ij}}=1$ if $c_{\mathrm{ij}} \geq \alpha_{j}$ and $b c_{\mathrm{ij}}=0$ otherwise. $\alpha_{j}$ is the threshold value of key attribute $\mathrm{j}$. We can also obtain the weight of each attribute as a priority of corresponding key attribute for customers view $w_{j}^{c}$ using the formula (1).

$w_{j}{ }^{c}=\frac{\sum_{i} b c_{i j}}{\sum_{i} \sum_{j} b c_{i j}} \quad \forall j$

With this weight we modify the CKIM matrix to obtain the customer profile matrix as given in formula (2).

$M C K I M=C K I M \times\left\lfloor w_{j}^{c}\right\rfloor$

Where $\left[w_{j}^{c}\right]=\left[\begin{array}{ccccc}w^{c}{ }_{1} & 0 & 0 & \cdots & 0 \\ 0 & w^{c}{ }_{2} & 0 & \cdots & 0 \\ \cdot & & & & \cdot \\ \cdot & & & & \cdot \\ \cdot & & & & \cdot \\ 0 & 0 & 0 & \cdots & w_{n}^{c}\end{array}\right]$

\section{Supplier Profile}

The agent forwards all information related to each product in the customer profile to all suppliers electronically. Then agent obtains the fuzzy value of each supplier on key attributes of customers. By obtaining all information from supplier-side we create the supplier-key attribute incidence matrix. The agent designs the supplier-key attribute incidence matrix as $S K I M=\left[s_{\mathrm{ij}}\right]$, where $s_{i j}(i=1,2, \ldots, m$ and $j=1,2, \ldots, n)$ represents the fuzzy value of $m$ suppliers on corresponding attributes. This value indicates the priority of suppliers to satisfy the specific attribute of customers.

On the basis of a specified threshold value for each key attribute, the above matrix could be converted into a binary matrix $B S K I M=\left[b s_{\mathrm{ij}}\right]$, where $b s_{\mathrm{ij}}=1$ if $s_{\mathrm{ij}} \geq \beta_{j}, \quad$ and $b s_{\mathrm{ij}}$ $=0$ otherwise. $\beta_{j}$ is the threshold value of the key attribute $j$. Then we obtain the supplier's weight or priority of each key attribute $w_{j}{ }^{s}$ as shown in the formula (3).

${ }^{1}$. The threshold value has been considered as a minimum expectation value to purify the reliability of the fuzzy value. It could be dependent on internal and external factors. 
$w_{j}^{s}=\frac{\sum_{i} b s_{i j}}{\sum_{i} \sum_{j} b s_{i j}} \quad \forall j$

With this weight we modify the SKIM matrix to obtain a supplier profile matrix $M S K I M$ as shown in formula (4).

$$
\begin{aligned}
& M S K I M=S K I M \times\left\lfloor w_{j}{ }^{s}\right\rfloor \\
& \text { Where }\left[w_{j}{ }^{s}\right]=\left[\begin{array}{ccccc}
w_{1}{ }_{1} & 0 & 0 & \cdots & 0 \\
0 & w^{s}{ }_{2} & 0 & \cdots & 0 \\
\cdot & & & & \cdot \\
\cdot & & & & \cdot \\
\cdot & & & & \cdot \\
0 & 0 & 0 & \cdots & w_{n}^{s}
\end{array}\right]
\end{aligned}
$$

\section{Supply Chain Profile}

The match between supplier and buyer are obtained by combining customer profile matrix and supplier profile matrix using formula (5).

$$
M S C=M C K I M \times M S K I M^{T}=\left[s c_{i j}\right]
$$

The above matrix introduces the fuzzy priority of matching between sellers and customers in an electronic supply chain environment. For a match to be meaningful, the level of priority should be high enough, or higher than a threshold. On the basis of a specified threshold value we can convert the above matrix into a modified matrix $M M S C$ $=\left[b s c_{\mathrm{ij}}\right]$, where $b s c_{\mathrm{ij}}=1$ if $s c_{\mathrm{ij}} \geq \theta$, otherwise $b s c_{\mathrm{ij}}=0 . \theta$ is the threshold value.

\section{Feasibility Analyzer}

Actual managerial decisions are made both based on preference priority and practical feasibility. With feasibility analyzer considering the feasibility structure, we convert the $M M S C$ matrix to a Decision Matrix Supply Chain $D M S C=\left[d s c_{\mathrm{ij}}\right]$, where $d s c_{\mathrm{ij}}$ $=1$ if $b s c_{\mathrm{ij}}=1$ and feasible, otherwise $d s c_{\mathrm{ij}}=0$. Then we can use this matrix for the transportation model as illustrated in the next section.

\section{TRANSPORTATION MODEL}

Assume that suppliers are located in different nations with a vast network of clearing and forwarding agents. The integration of these geographically separated supplier locations and the fulfillment of the demands of different customer centers are a big challenge. A decision matrix can be developed based on a transportation model to allocate the distribution plans to different supplier locations, with the objective of 
minimizing the total cost. Further, the influence of non-quantifiable factors can also be included in the cost matrix. The influence of such factors can be reflected by incorporating the relative importance with respect to a given combination of supplierbuyer location. The total cost includes both the distribution cost and the inventory carrying cost.

In the present problem, we consider the demand of a family of products for the planning period that is obtained by the agent. The information for a rough-cut capacity planning will be carried out at different supplier locations based on actual shift time, total actual time available during the planning period, and the average break-down by supplier-agent interaction. The actual cost elements are represented in the form of a transportation matrix as $[T]=C_{i j r s}$, where:

$n b:$ the number of buyers

$\mathrm{nm}:$ the number of suppliers

$n p:$ the number of periods.

Let $C_{i j r s}$ be the cost for supplying one unit of a family of products to a buyer location $i$ in the period $r$ from supplier location $j$ distributed during the period $s$ for $r \geq s$, otherwise the value will take infinity. $C_{i j r s}$ includes both transportation and inventory costs.

$i=1,2, \ldots, n b ; j=1,2, \ldots, n m ; r=1,2, \ldots, n p ;$ and $s=1,2, \ldots, n p$.

The transportation model with actual cost elements $C_{i j r s}$ is given in Table 2.

\section{Determination of Supplier-Buyer Mix}

The final priority of each supplier-buyer location can be determined by performing the matrix multiplication of the supplier and buyer location priority as shown in Table 3 with $a_{i j}$ values $(i=1, \ldots, n b, j=1, \ldots, n m)$.

This matrix has been illustrated as an SC profile with $a_{i j}=0$, if $d s c_{\mathrm{ij}}=0$ and there is no feasibility to match between supplier $j$ and buyer $i$. 
Table2 Transportation Model with Actual Cost Elements

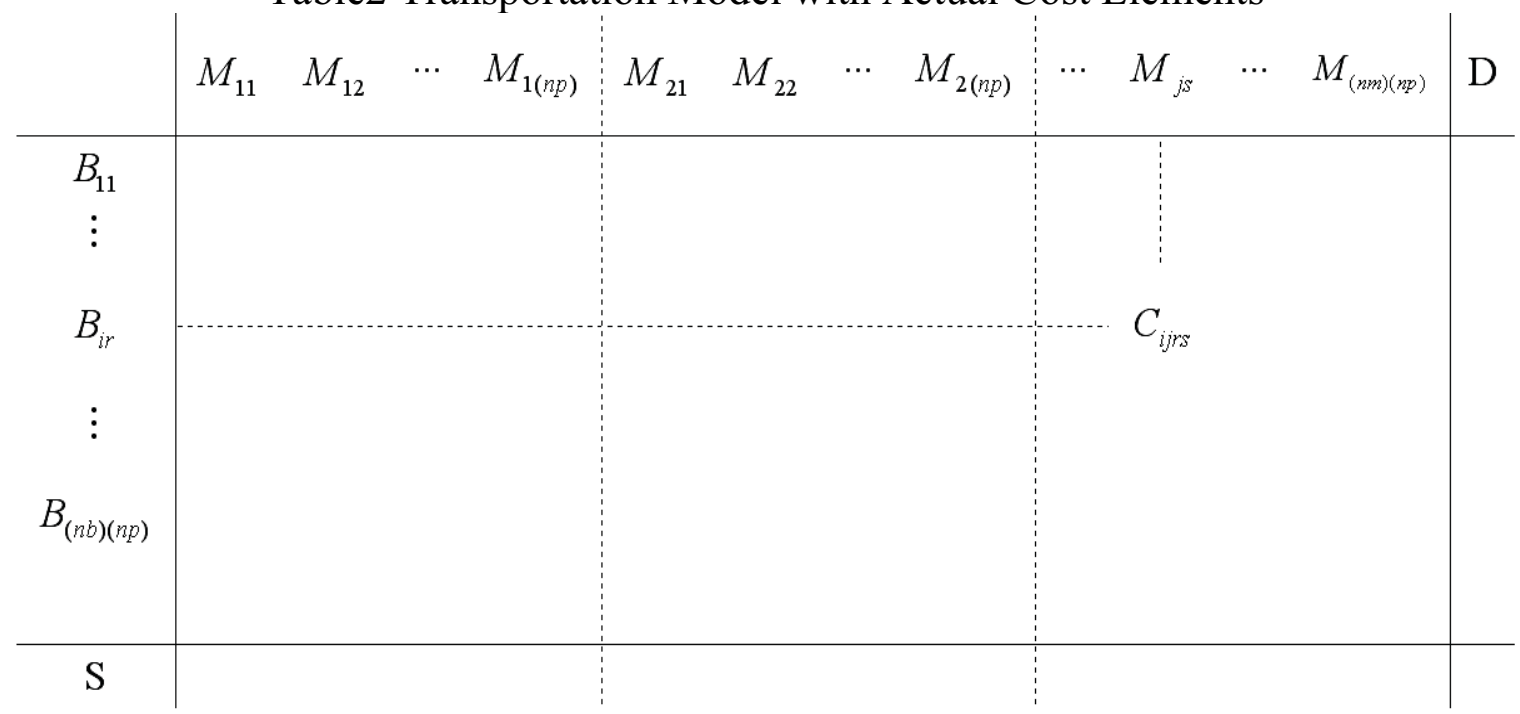

Where demands (D) obtained from the agent for various customers in defined periods and the supply quantities (S) have been shown at supplier location at different periods.

$B_{i r}$ Indicates customer location $i$ at period $r$ and $M_{j s}$ stands for supplier location $j$ at period $s$.

Table3 [BM] Overall Priority Matrix of Supplying a Family of Product to a Customer $(B)$ from A Particular Seller Location $(M)$

\begin{tabular}{c|ccccc} 
& $M_{1}$ & $M_{2}$ & $\ldots$ & $M_{(n m)}$ \\
\hline$B_{1}$ & $a_{11}$ & $a_{12}$ & $\ldots$ & $a_{1(n m)}$ \\
$B_{2}$ & $a_{21}$ & $a_{22}$ & $\ldots$ & $a_{2(n m)}$ \\
$\ldots$ & $\ldots$ & $\ldots$ & & $\ldots$ \\
$B_{(n b)}$ & $a_{(n b) 1}$ & $a_{(n b) 2}$ & $\ldots$ & $a_{(n b)(n m)}$ \\
\hline
\end{tabular}

\section{Development of the Transportation Matrix}

It is evident that, based on the association between the elements of matrix [BM] and cost elements, the lower the probability of shipping from a supplier location to a customer, the higher the costs will be. Hence, we have to modify the elements of Table 3 as:

$\left[B M^{\prime}\right]=1-[B M]$

So that $a_{i j}^{\prime}=1-a_{i j}$ for all entities of the corresponding matrix. If $a_{i j}=0$, then $a_{i j}^{\prime}$ will take infinity value. This modification produces a matrix that represent the nondesirability of shipping from a supplier location to a given customer location as given in 
Table 4. Thus, this matrix becomes compatible with the transportation matrix and can be directly incorporated.

Table4 [BM'] Non-Desirability Matrix of Supplying a Family of Product to a Customer from a Particular Supplier Location

\begin{tabular}{c|ccccc} 
& $M_{1}$ & $M_{2}$ & $\ldots$ & $M_{(n m)}$ \\
\hline$B_{1}$ & $1-a_{11}$ & $1-a_{12}$ & $\ldots$ & $1-a_{1(n m)}$ \\
$B_{2}$ & $1-a_{21}$ & $1-a_{22}$ & $\ldots$ & $1-a_{2(n m)}$ \\
$\ldots$ & $\ldots$ & $\ldots$ & & $\ldots$ \\
$B_{(n b)}$ & $1-a_{(n b) 1}$ & $1-a_{(n b) 2}$ & $\ldots$ & $1-a_{(n b)(n m)}$ \\
\hline
\end{tabular}

Finally, total costs should be adjusted to reflect non-quantifiable factors. The transportation model with modified total cost elements reflecting qualitative factors can be obtained as $\left[T^{\prime}\right]=C_{i j r s}^{\prime}$, so that

$$
C_{i j r s}^{\prime}=\left(1-a_{i j}\right) C_{i j r s} .
$$

Using Lingo package to solve the above transportation matrix, the final optimum of allocation can be obtained. We can then apply the following evolutionary rule for an automatic evolution of the structure of supply chains in a dynamic fashion.

\section{EVOLUTIONARY ALGORITHM}

We consider the following steps as evolutionary rule to redesign the SC structure.

Step0. $\mathrm{T}=0$.

Step1. Obtain the customer, supplier and supplier-customer profiles using transaction agent (TA).

Step2. Apply the distribution policy using the developed transportation matrix as shown in the transportation model.

Step3. Assign the quantity of material flows and update the information flows.

Step4. $\mathrm{T}=\mathrm{T}+1$.

Step5. Update the SC network and go to step1.

\section{CONCLUSION}

Due to the diversity in the functionality and complexity of supply chain systems, we have introduced the concept of dynamic supply chain information flows into e-SCM. An agent is designed to facilitate the preprocessing of customer attribute values as well as 
supplier views. The basic information is obtained in the form of customer-key attribute incidence matrix to achieve real-time customer profile. The supplier profile is designed to analyze the possibility of interaction between two major actors in SC, suppliers and buyers. The interaction between two profiles is computationally derived to produce the SC profile. This profile is used to develop a practical transportation model with nonquantitative parameters such that mass-customized service is reflected.

In this paper, we theorized major aspects of information flows between suppliers and buyers. On the basis of the information flow and preprocessing of data, buyersupplier relationships are represented so that it can also incorporate non-quantitative parameters. The priority match can be examined and used for the logistical management of transportation model. This approach presents a great potential to resolve several problems in real-world SC systems which are in conflict with each other. This research provides a model of reliable and dynamic structure of e-SCM that can improve supply chain performance based on the fuzzy logic. To understand the effects of the variations in the threshold values, $\alpha_{j}, \beta_{j}$ and $\theta$, sensitivity analyses can be performed and modified weights for corresponding profiles can be obtained. The use of current conceptual model for an extensive empirical analysis will lead to an interesting area of further research.

\section{ACKNOWLEDGMENTS}

The authors acknowledge the editorial contribution of the research assistants Jiho Son, Heeyoun Kim, Lynn Kim, and Jingyoo Cho at the Digital Business and Management Center of Hanyang University, Seoul, Korea.

\section{REFERENCES}

Fazel Zarandi, M.H. Turksen, I.B. and Saghiri, S. 2002. Supply Chain: Crisp and Fuzzy Aspects, Int. J. Appl. Math. Comput. Sci., 12(3), 423-435.

Flynn, B.B. and Flynn, E.J. 2005. Synergies between supply chain management and quality management: emerging implications, International Journal of Production Research, 43(16), 3421-3436.

Lee, H.L. 2004. The triple-A supply chain. Harvard Business Review, 82(10), 102-112.

Liker, J.K. and Choi, T.Y. 2004. Building deep supplier relationships, Harvard Business Review, 82(12), 104-113.

Mehra, S. and Agrawal, S.P. 2003. Total quality as a new global competitive strategy, Int. J. Quality and Reliability Manage., 20(8/9), 1009-1025. 
Mehra, S. and Inman, R.A. 2004. Purchasing management and business competitiveness in the coming decade. Prod. Planning and Control, 15(7), 710-718.

Meredith, J. and Roth, A.1998. Operations management in the USA, Int. J. Op. and Prod. Manage., 18(7), 668-683.

Narayanan, V.G. and Raman, A. 2004. Aligning incentives in supply chains, Harvard Business Review, 82(11), 94-102.

Poulymenakou, A. and Tsironis, L. 2003. Quality and electronic commerce: a partnership for growth, The TQM Magazine, 15(3), 137-151.

Swaminathan, J.M. and Tayur, S.R., 2003. Models for Supply Chains in E-Business, Management Science, 49(10), 1387-1406. 
